\title{
Effects of almond consumption on the post-lunch dip and long-term cognitive function in energy-restricted overweight and obese adults
}

\author{
Jaapna Dhillon, Sze-Yen Tan and Richard D. Mattes* \\ Department of Nutrition Science, Purdue University, West Lafayette, IN 47907, USA \\ (Submitted 22 June 2016 - Final revision received 7 November 2016 - Accepted 7 December 2016 - First published online 10 February 2017)
}

\begin{abstract}
The post-lunch dip in cognition is a well-established phenomenon of decreased alertness, memory and vigilance after lunch consumption. Lunch composition reportedly influences the post-lunch dip. Moreover, dieting is associated with cognitive function impairments. The negative effects of dieting have been reversed with nut-supplemented diets. The aims of this study were to (1) evaluate the acute effect of an almond-enriched high-fat lunch or high-carbohydrate lunch on the post-lunch decline in cognitive function, and (2) evaluate the effects of chronic almond consumption as part of an energy-restricted diet on the memory and attention domains of cognitive function. In total, eightysix overweight and obese adults were randomised to consume either an almond-enriched diet (AED) or a nut-free control diet (NFD) over a 12-week weight loss intervention. Participants were also randomised to receive either an almond-enriched high-fat lunch (A-HFL) ( $>55 \%$ energy from fat, almonds contributing $70-75 \%$ energy) or a high-carbohydrate lunch (HCL) (>85\% energy from carbohydrates) at the beginning and end of the weight loss intervention. Memory and attention performance indices decreased after lunch consumption $(P<0 \cdot 001)$. The A-HFL group ameliorated the decline in memory scores by $57.7 \%$ compared with the HCL group $(P=0 \cdot 004)$. Both lunch groups had similar declines in attention. Moreover, memory and attention performance indices increased after the 12 -week intervention period $(P<0.05)$ with no difference between the AED and NFD groups. In conclusion, almond consumption at a midday meal can reduce the post-lunch dip in memory. However, long-term almond consumption may not further improve cognitive function outcomes in a weight loss intervention.
\end{abstract}

\section{Key words: Cognitive function: Post-lunch dip: Almonds: Nuts: Energy restriction}

The post-lunch dip in cognitive performance is a well-established phenomenon of decreased cognitive function in the early afternoon hours typically after lunch consumption ${ }^{(1)}$. It is thought to begin approximately $1 \mathrm{~h}$ after the start of lunch consumption. During the post-lunch dip, memory and vigilance are the most severely affected domains, and decreased mood, alertness and anxiety are also reported ${ }^{(2,3)}$. These symptoms may negatively affect cognitive performance and increase personal and societal risks ${ }^{(1,4,5)}$. Lunch composition is reported to play a role in the post-lunch dip with high-fat lunches leading to slower ${ }^{(6,7)}$ but more accurate ${ }^{(7)}$ responses than low-fat lunches. Highcarbohydrate meals reportedly have a sedative effect promoting drowsiness in females and calmness in males ${ }^{(8)}$. There is heterogeneity to these responses in terms of age. For example, adults $\geq 40$ years show impaired cognitive performance after a high-carbohydrate lunch, whereas adults $<40$ years do not ${ }^{(8)}$. However, in healthy young adults, tasks that demand greater cognitive function are more sensitive to nutrient manipulations ${ }^{(9)}$. The unique nutrient profile of almonds, which is lower in carbohydrate and high in unsaturated fats, may lessen this post-lunch dip in young and middle-aged adults. If almonds are able to ameliorate the post-lunch dip, they can enhance productivity (e.g. accuracy of written tasks) and safety (staying awake while driving or working with machines).

Nut consumption over the long term is also positively associated with cognitive performance ${ }^{(10-12)}$. The beneficial effects in the long term are likely due to the improvements in cardiovascular risk factors such as lipid profile, arterial compliance, glucoregulation, oxidative stress, blood pressure and inflammation, with subsequent improvements in endothelial function and cerebral vascular function ${ }^{(13)}$. However, there is very limited data from interventional trials in humans to establish causation.

The process of dieting to lose weight is associated with cognitive function impairments ${ }^{(14)}$ as well that could be a result of food restriction, anxiety related to maintaining a weight loss regimen or preoccupying thoughts of hunger and desire to eat ${ }^{(15)}$ and body esteem $^{(16,17)}$. Negative effects of dieting on cognitive performance have been reversed with nut-supplemented diets higher in fat and lower in carbohydrate ${ }^{(18)}$. Hence, learning and memory may also be enhanced with the inclusion of almonds during weight loss.

Abbreviations: AED, almond-enriched diet; A-HFL, almond-enriched high-fat lunch group; CP, concentration performance; HCL, high-carbohydrate lunch group; NFD, nut-free diet; TN, quantitative performance; TNE, qualitative performance; VLR, verbal list recognition.

* Corresponding author: R. D. Mattes, email mattes@purdue.edu 
The purpose of this study was 2 -fold. The first aim was to determine the post-lunch changes in cognitive function with almond consumption at lunch compared with a highcarbohydrate control lunch. We hypothesised that inclusion of almonds in a midday meal will ameliorate the post-lunch decline in memory and attention compared with a high carbohydrate control meal by lowering the percentage of dietary energy from carbohydrates and increasing the percentage of energy from fat. The second aim was to evaluate the effects of almond consumption as part of an energy-restricted diet on cognitive function compared with a nut-free diet (NFD) matched on energy restriction (control). We hypothesised that inclusion of almonds in an energy-restricted diet will improve the attention and memory domains of cognitive function compared with the control.

\section{Methods}

\section{Participants}

In total, eighty-six healthy overweight or obese adults (twentyone men and sixty-five women) participating in an almond weight loss clinical trial were recruited ${ }^{(19)}$. The eligibility criteria were as follows: age of $18-60$ years, BMI of $25-40 \mathrm{~kg} / \mathrm{m}^{2}$, no nut allergies, willingness to consume study foods and comply with the study protocol, no endocrine or metabolic disorders, non-smokers, and consistent diet and activity patterns. This study was conducted according to the guidelines codified in the Declaration of Helsinki and is registered on ClinicalTrials.gov (registration no. NCT02360787). All procedures involving human subjects were approved by the Purdue Institutional Review Board. Participants were recruited via public advertisements. Those meeting the eligibility criteria were contacted to schedule a screening visit. Participants provided written consent at the beginning of the screening session and were compensated for their time in the study.

\section{Intervention}

The study was a 12-week randomised, controlled, parallel-arm weight loss trial ${ }^{(19)}$. In brief, participants were randomised into one of two energy-restricted study arms: almond-enriched diet
(AED) or NFD groups (Fig. 1). Both groups received dietary counselling to reduce energy intake to achieve $2092 \mathrm{~kJ} / \mathrm{d}$ $(500 \mathrm{kcal} / \mathrm{d})$ deficits to support weight loss. Weekly energy and nutrient analyses using 24-h food recalls were conducted to determine the participants' compliance to the dietary recommendations ${ }^{(19)}$.

In total, forty-three participants were randomised into each of the AED and NFD groups. Participants in the AED group were asked to consume dry-roasted, lightly salted almonds providing $15 \%$ of the energy in their individualised energy-restricted diet. Energy from almonds was accounted for during dietary modelling so that a $2092 \mathrm{~kJ} / \mathrm{d}(500 \mathrm{kcal} / \mathrm{d})$ deficit was achieved. Participants in the NFD group were asked to avoid all nuts and nut products during the intervention period. Cognitive function outcomes were assessed at the beginning (baseline) and end of the 12-week intervention.

\section{Test meals for post-lunch dip assessment}

Participants were randomised to either an almond-enriched high-fat lunch group (A-HFL) or a high-carbohydrate lunch group (HCL) at the beginning of the study (Fig. 1). The lunch provided $23-25 \%$ of the participants' estimated daily energy intake. Their individualised energy intake at lunch was determined from the What We Eat in America, National Health and Nutrition Examination Survey (NHANES) 2011-2012 survey that reports the percentage of energy intake at lunch according to sex and age ${ }^{(20)}$. Participants in the HCL meal were fed a combination of spaghetti in tomato sauce (SpaghettiOs), white bread, jelly and apple juice providing greater than $85 \%$ energy from carbohydrate. Participants in the A-HFL meal were fed almonds with some SpaghettiOs and/or white bread, jelly and apple juice providing greater than $55 \%$ energy from fat. The almonds provided $70-75 \%$ energy in the A-HFL meal. An example of a sample lunch is provided in Table 1. Participants were instructed to eat the entire test meal within $15 \mathrm{~min}$. Cognitive function outcomes were assessed immediately after lunch and 30-35 min later. In addition, the post-lunch dip assessments were performed at baseline and at the end of the 12 -week intervention.

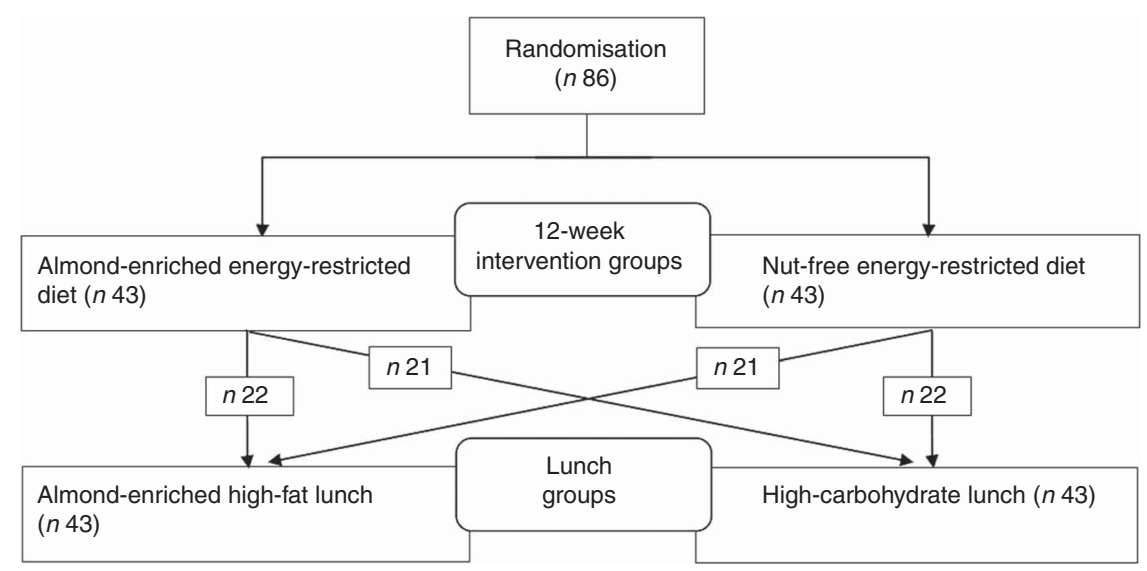

Fig. 1. Participant randomisation to the 12-week intervention and lunch groups. 
Table 1. Nutrient composition of a sample lunch provided to a 28-year-old male participant on a $7431.2 \mathrm{~kJ} / \mathrm{d}(1800 \mathrm{kcal} / \mathrm{d})$ diet in both lunch groups

\begin{tabular}{|c|c|c|}
\hline & $\begin{array}{l}\text { Almond-enriched } \\
\text { high-fat lunch }\end{array}$ & $\begin{array}{l}\text { High-carbohydrate } \\
\text { lunch }\end{array}$ \\
\hline Food items* & $\begin{array}{l}53 \mathrm{~g} \text { almonds ( } 75 \% \\
\text { energy of lunch) } \\
\text { One-third } 12 \mathrm{oz} . \text { can } \\
\text { SpaghettiOs } \\
1 \text { cup water }\end{array}$ & $\begin{array}{l}\text { Two-thirds } 12 \text { oz. can } \\
\text { SpaghettiOs } \\
1 \text { slice white bread }(25 \mathrm{~g}) \\
1 \text { tablespoon jelly } \\
\text { Half cup apple juice } \\
1 \text { cup water }\end{array}$ \\
\hline $\begin{array}{l}\text { Energy (\% daily } \\
\text { intake) } \dagger\end{array}$ & 26 & 26 \\
\hline Carbohydrate (\%) & 31 & 86 \\
\hline Fat $(\%)$ & 56 & 4 \\
\hline Protein (\%) & 13 & 10 \\
\hline
\end{tabular}

* The study was not a crossover design, but this is exemplary of two meals for participants with similar energy intake levels.

† Calculated from the 'What We Eat in America survey'(20).

\section{Cognitive function test protocol}

The cognitive function test protocol is shown in Fig. 2. Participants arrived at the laboratory in a fasting state for measurement of other variables pertaining to the almond weight loss study such as body weight, body fat, blood pressure, serum insulin and glucose, etc. ${ }^{(19)}$. Instead of assessing cognitive outcomes before lunch in the fasted state, we assessed cognitive performance immediately after lunch in lieu of the pre-lunch time point as there is some evidence that skipping breakfast is associated with negative cognitive consequences ${ }^{(21)}$.

After measurement of the aforementioned variables, participants were provided with a midday meal according to their randomised lunch group (A-HFD or HCL). They were given $15 \mathrm{~min}$ to eat their meal after which they were taken into a quiet room for cognitive function testing. Testing included memory and attention domains of cognitive function. The tests for memory included the following: immediate memory, delayed memory and verbal list recognition (VLR) tests were adapted from the Repeated Battery for the Assessment of Neuropsychological Status tests ${ }^{(22)}$, whereas attention was assessed using the ' $\mathrm{d} 2$ ' test of attention ${ }^{(23)}$, respectively.

In the immediate memory test, participants were read a list of ten words immediately after lunch before the onset of the post-lunch dip. They were asked to remember and recall the words after $2 \mathrm{~min}$. The number of correct responses was recorded. In the delayed memory test, participants were asked to recall the same ten words read to them and record them on a sheet of paper $34 \mathrm{~min}$ after lunch consumption. The number of correct responses was tallied.

In the attention test, participants were asked to cross out the letter $\mathrm{d}$ accompanied with two dashes (above the letter $\mathrm{d}$, below the letter $\mathrm{d}$ or one dash above and one below the letter $\mathrm{d}$ ) on a recording blank with fourteen lines of forty-seven letters comprised of either $\mathrm{d}$ or $\mathrm{p}$ with one, two, three or four dashes. They were given $4 \mathrm{~min}$ to do this task. The errors of omission (skipping target d's) and errors of commission (marking inappropriate targets) were recorded. This test was used to assess quantitative

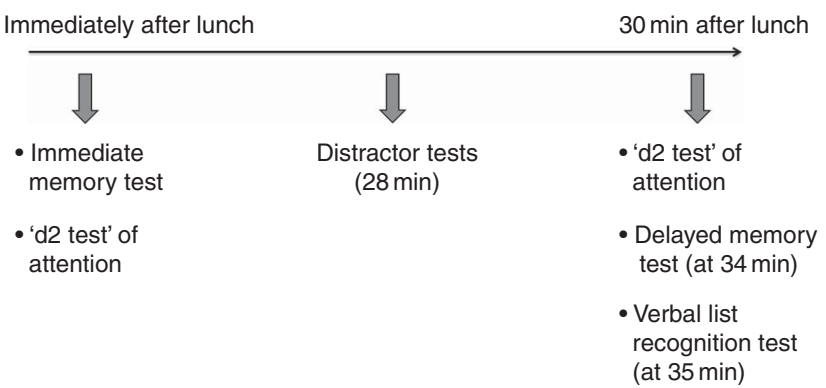

Fig. 2. Cognitive function test protocol at the beginning and end of the 12-week intervention.

performance (the total number of items processed, TN), qualitative performance (the total number of items processed minus total errors, TNE) and concentration performance (the total number of correct items marked minus errors of commission, CP). The attention tests were conducted immediately and 30 min after lunch.

Finally, the VLR test was used to assess the participant's ability to recall the ten words read out to them immediately after lunch. The tester read out a list of twenty words and participants had to acknowledge verbally which words were present in the initial list. The correct responses were marked.

Distractor tests adapted from the ' $\mathrm{d} 2$ test' of attention were used to fill in the 30-min interval after lunch to ensure that participants did not fall asleep. Participants were asked to cross out different combinations of irrelevant letters with dashes over $28 \mathrm{~min}$. These tests were not graded.

\section{Statistical analysis}

A linear mixed model analysis was performed on the correct responses obtained from the immediate and delayed memory tests, and the outcomes from the ' $\mathrm{d} 2$ ' test of attention (i.e. concentration performance, quantitative performance and qualitative performance. The intervention period (before $v$. after) and the lunch period (immediately after $v .35 \mathrm{~min}$ after) were used as within-subject factors and the intervention and lunch groups as between-subject factors. The correct responses from the VLR test were analysed using a linear mixed model with intervention period as the within-subject factor and the intervention groups as between-subject factors. An additional linear mixed model analysis on the change in outcomes as opposed to absolute values was also performed. Age, sex and BMI were also considered as between-subject factors for all the tests. When significant interactions were observed, pair-wise comparisons were carried out with Bonferroni correction.

Between-group differences were assessed at baseline using independent-samples $t$ tests. Pearson's statistics were used to determine correlations. The $\alpha$ level was set at $0 \cdot 05$. SPSS (version 22, 2013; SPSS Inc.) was used for all the statistical analyses. OriginPro (version b9.3.226, 2016; OriginPro Corporation) was used to graph the data. Data are reported as means with their standard errors unless otherwise stated. The sample size calculations for this study were based on visceral fat, which was one of the primary outcomes for the almond 
weight loss study ${ }^{(19)}$. However, analysis of the current data revealed that the study could detect the following effect sizes for the cognitive function outcomes over the 12-week intervention with $80 \%$ power (memory - 0.9, CP $-13 \cdot 1$, TN $-25 \cdot 9$, TNE - 30).

\section{Results \\ Participants}

The baseline characteristics of all participants are reported in Table 2. Of the eighty-six participants who were enrolled in the study, seven withdrew during the intervention. Four participants withdrew due to non-compliance with the dietary protocol, one withdrew due to illness not related to the study, one female participant withdrew because of pregnancy and one withdrew due to lack of time to devote to the study. There were no significant differences in attrition between the AED and NFD intervention groups at 12 weeks.

\section{Compliance to intervention}

Total energy intake decreased (from baseline) in both AED $(-976.17$ (SEM 427.98) kJ) and NFD groups (-1222.9) (SEM $439.7) \mathrm{kJ})$ during the energy restriction period $(P<0.05)^{(19)}$. The percentages of energy from fat $(36.62$ (SEM 1.42) v. 29.36 (SEM $1.46) \%$ ) (AED $v$. NFD) and total MUFA (27.70 (SEM 1.43) $v .15 .43$ (SEM 1.47) g), total $\alpha$-tocopherol (15.15 (SEM 0.8) v. 6.43 (SEM $0 \cdot 82) \mathrm{mg}$ ), $\mathrm{Mg}(311.98$ (SEM 16.85) v. $245 \cdot 36$ (SEm 17.31) mg) and phytic acid (850.86 (SEM 64.13) v. 614.42 (SEM 65.89) $\mathrm{mg}$ ) intake were greater, and the percentage of energy from carbohydrate (46.34 (SEM 1.65) v. 51.93 (SEM 1.70) \%) was lower in the AED group compared with the NFD group at the end of the intervention $(P<0.05)$. These nutrient intake patterns reflect the nature of the dietary intervention ${ }^{(19)}$.

\section{Cognitive function outcomes}

Memory outcomes. Memory scores did not differ between the A-HFL and HCL meals immediately after lunch. However, memory scores decreased significantly 35 min after consumption of lunch in both groups $(P<0 \cdot 001)$. The A-HFL meal ameliorated the decline in memory scores by $57.7 \%$ compared with the HCL meal ( $P=0.004)$ (Fig. 3).

In addition, there were no significant differences between the mean number of correct words recorded immediately after lunch (immediate memory score) and mean number of correct words recorded $35 \mathrm{~min}$ after lunch (delayed memory score) between the AED and NFD groups at baseline. Both memory scores increased significantly after the 12-week weight loss intervention period $(P<0 \cdot 001)$, but the difference between the groups was not significant (Fig. 4).

Verbal list recognition outcomes. There were no significant differences between the mean number of words correctly recognised verbally (VLR score) between the AED and NFD groups at baseline. Although the VLR score increased after the 12-week weight loss intervention in both groups (AED: 0.47

Table 2. Baseline characteristics of participants

(Mean values and standard deviations; numbers and percentages)

\begin{tabular}{|c|c|c|c|c|c|c|c|c|}
\hline & \multicolumn{4}{|c|}{ 12-week intervention groups } & \multicolumn{4}{|c|}{ Post-lunch dip experimental groups } \\
\hline & \multicolumn{2}{|c|}{ AED $(n 43)$} & \multicolumn{2}{|c|}{ NFD $(n 43)$} & \multicolumn{2}{|c|}{ A-HFL ( $n$ 43) } & \multicolumn{2}{|c|}{$\mathrm{HCL}(n$ 43) } \\
\hline & Mean & SD & Mean & SD & Mean & SD & Mean & SD \\
\hline Age (years) & 31.05 & $12 \cdot 9$ & $31 \cdot 77$ & $13 \cdot 15$ & $30 \cdot 30$ & $12 \cdot 89$ & 32.51 & 13.08 \\
\hline Body weight (kg) & $82 \cdot 79$ & $12 \cdot 9$ & $84 \cdot 71$ & $14 \cdot 12$ & $84 \cdot 38$ & 14.50 & $83 \cdot 12$ & $12 \cdot 51$ \\
\hline \multirow[t]{2}{*}{ BMI $\left(\mathrm{kg} / \mathrm{m}^{2}\right)$} & $29 \cdot 91$ & $3 \cdot 20$ & $30 \cdot 97$ & $4 \cdot 48$ & $30 \cdot 93$ & $4 \cdot 42$ & $29 \cdot 94$ & $3 \cdot 29$ \\
\hline & $n$ & $\%$ & $n$ & $\%$ & $n$ & $\%$ & $n$ & $\%$ \\
\hline \multicolumn{9}{|l|}{ Sex } \\
\hline Male & 11 & $25 \cdot 6$ & 10 & $23 \cdot 3$ & 9 & $20 \cdot 9$ & 12 & $27 \cdot 9$ \\
\hline Female & 32 & 74.4 & 33 & $76 \cdot 7$ & 34 & $79 \cdot 1$ & 31 & $72 \cdot 1$ \\
\hline \multicolumn{9}{|l|}{ Age range } \\
\hline 18-39 years & 32 & 74.4 & 31 & $72 \cdot 1$ & 33 & $76 \cdot 7$ & 30 & $69 \cdot 7$ \\
\hline 40-49 years & 5 & 11.6 & 4 & $9 \cdot 3$ & 3 & $7 \cdot 0$ & 6 & $14 \cdot 0$ \\
\hline $50-60$ years & 6 & $14 \cdot 0$ & 8 & $18 \cdot 6$ & 7 & $16 \cdot 3$ & 7 & $16 \cdot 3$ \\
\hline \multicolumn{9}{|l|}{ Weight category } \\
\hline Overweight (BMI 25-29.9 kg/m²) & 23 & 53.5 & 21 & $48 \cdot 8$ & 21 & $48 \cdot 8$ & 23 & $53 \cdot 5$ \\
\hline Obese (BMI 30-40 kg/m²) & 20 & $46 \cdot 5$ & 22 & 51.5 & 22 & $51 \cdot 2$ & 20 & $46 \cdot 5$ \\
\hline \multicolumn{9}{|l|}{ Cognitive function scores* } \\
\hline Memory & $6 \cdot 1$ & 1.5 & $6 \cdot 0$ & 1.5 & $6 \cdot 1$ & 1.5 & $6 \cdot 0$ & 1.5 \\
\hline VLR & $18 \cdot 2$ & 1.6 & $17 \cdot 9$ & 1.6 & - & - & - & - \\
\hline Concentration performance & $165 \cdot 2$ & $32 \cdot 5$ & $155 \cdot 3$ & $28 \cdot 0$ & $163 \cdot 9$ & $34 \cdot 3$ & $156 \cdot 7$ & $26 \cdot 2$ \\
\hline Quantitative performance & $377 \cdot 2$ & $71 \cdot 8$ & $368 \cdot 2$ & $56 \cdot 8$ & $378 \cdot 7$ & 71.6 & $366 \cdot 7$ & $56 \cdot 8$ \\
\hline Qualitative performance & $371 \cdot 8$ & $71 \cdot 6$ & $356 \cdot 8$ & $56 \cdot 6$ & $371 \cdot 1$ & $72 \cdot 6$ & 357.5 & $55 \cdot 5$ \\
\hline
\end{tabular}

AED, almond-enriched diet; NFD, nut-free diet; A-HFL, almond-enriched high-fat lunch; HCL, high-carbohydrate lunch; VLR, verbal list recognition; -, not applicable.

* Cognitive function scores before the 12-week intervention for the AED and NFD intervention groups and cognitive function scores immediately after lunch for the A-HLF and HCL groups before the 12-week intervention. 


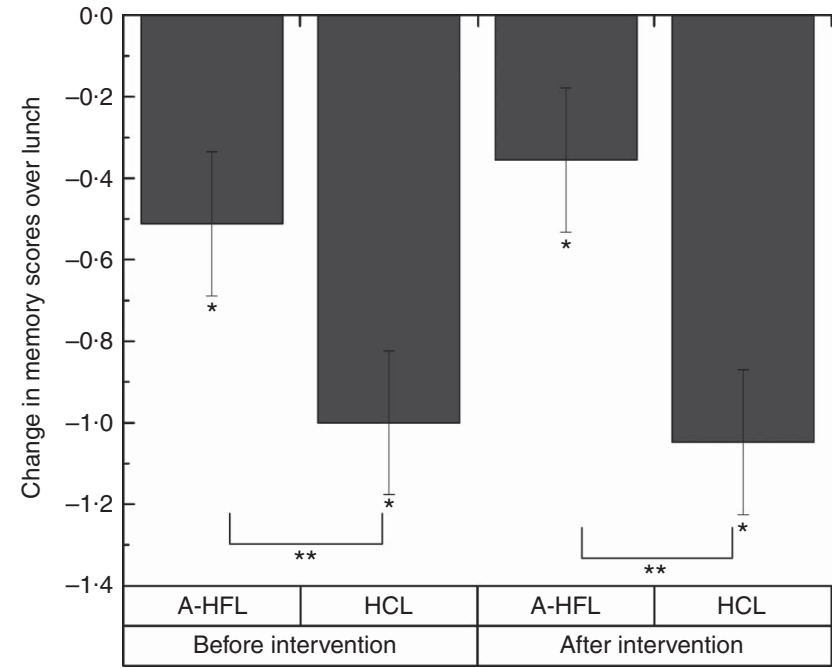

Fig. 3. Mean change in memory scores immediately after lunch and $35 \mathrm{~min}$ after lunch consumption in the almond-enriched high-fat lunch (A-HFL, $n$ 43) and high-carbohydrate lunch (HCL, $n 43$ ) groups before and after the 12-week intervention. Values are means, with their standard errors obtained from a linear mixed-effects model with lunch period and intervention period as within-subject factors and lunch group and intervention group as betweensubject factors. * Significant differences for change in memory scores over lunch period $(P<0.001) .{ }^{* *}$ Significantly different between the A-HFL and HCL groups $(P=0.004)$.

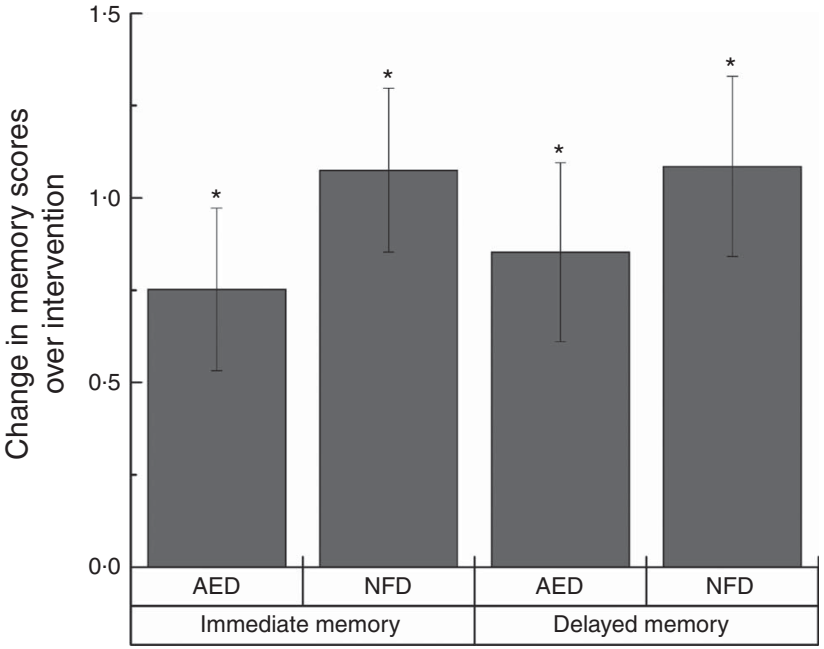

Fig. 4. Mean change in immediate and delayed memory scores before and after the 12-week intervention in the almond-enriched diet (AED, $n$ 43) and the nut-free diet (NFD, $n$ 43) groups. Values are means, with their standard errors obtained from a linear mixed-effects model with intervention period and lunch period as within-subject factors and intervention group and lunch group as between-subject factors. * Significant differences for change in memory scores over intervention period $(P<0.001)$

(sem 0.25), NFD: 0.63 (sem 0.25), number of words, $P=0.003$ ), the difference between the groups was not significant.

Attention outcomes. The performance indices, that is concentration performance (CP), quantitative performance (TN) and qualitative performance (TNE), did not differ between the A-HFL and HCL meals immediately after lunch. However, while

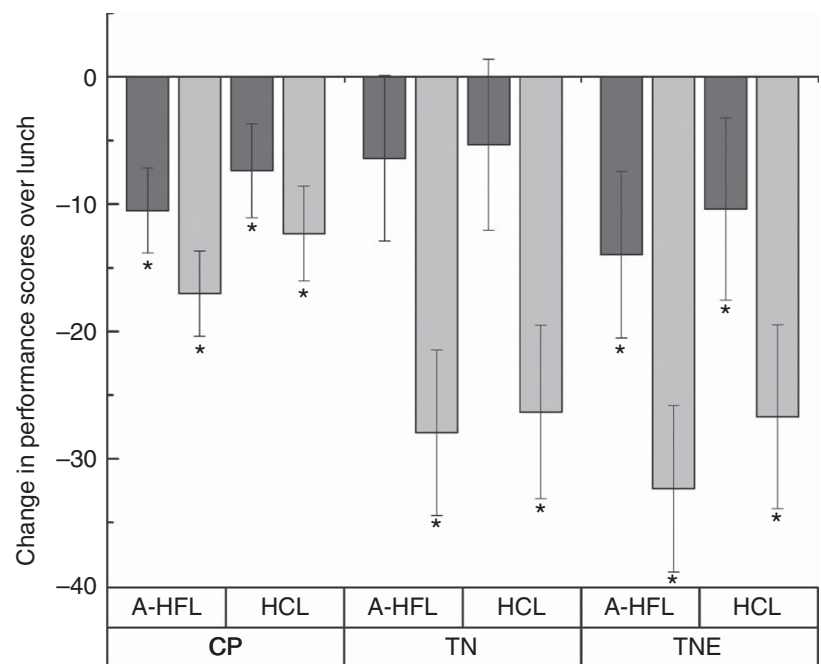

Fig. 5. Mean change in performance indices immediately after lunch and 35 min after lunch consumption in the almond-enriched high-fat lunch (A-HFL, $n$ 43) and high-carbohydrate lunch (HCL, $n$ 43) groups before and after the 12-week intervention. Values are means, with their standard errors obtained from a linear mixed-effects model with lunch period and intervention period as within-subject factors and lunch group and intervention group as betweensubject factors. * Significant differences for change in performance indices over the lunch period $(P<0.001)$. CP, concentration performance (total number of correct items marked minus errors of commission); $\mathrm{TN}$, quantitative performance (total number of items processed); TNE, qualitative performance (total number of items processed minus total errors). $\square$, Before intervention; $\square$, after intervention.

$\mathrm{CP}$ and TNE decreased significantly 35 min after consumption of lunch $(P<0.001)$ (Fig. 5), TN decreased significantly 35 min after consumption of lunch only after the weight loss intervention $(P=0.001)$ (Fig. 5). There were no significant differences in the performance indices between the AED and NFD groups at baseline. Although the performance indices increased after the weight loss intervention $(P<0.001)$, the difference between the groups was not significantly different (Fig. 6).

Participants aged 18-39 years had higher scores for all performance indices than participants aged 50-60 years regardless of intervention group and post-lunch dip group $(P<0 \cdot 05$, data not shown). Age was also moderately correlated with all performance indices regardless of group. The correlation coefficients between age and performance indices (CP, TN and TNE) ranged from -0.348 to $-0.487(P<0.05)$.

There was no effect of sex, weight category (BMI) or amount of weight loss on memory, VLR or attention outcomes. In addition, there were no acute lunch-chronic intervention interaction effects for the cognitive function outcomes.

\section{Discussion}

The present study confirmed the presence of a post-lunch dip in cognitive function. Almond consumption at lunch ameliorated the post-lunch decline in memory but not attention performance. In addition, cognitive function improved over the 12-week weight loss intervention period. However, almond consumption for 12 weeks did not further improve cognitive function outcomes. 


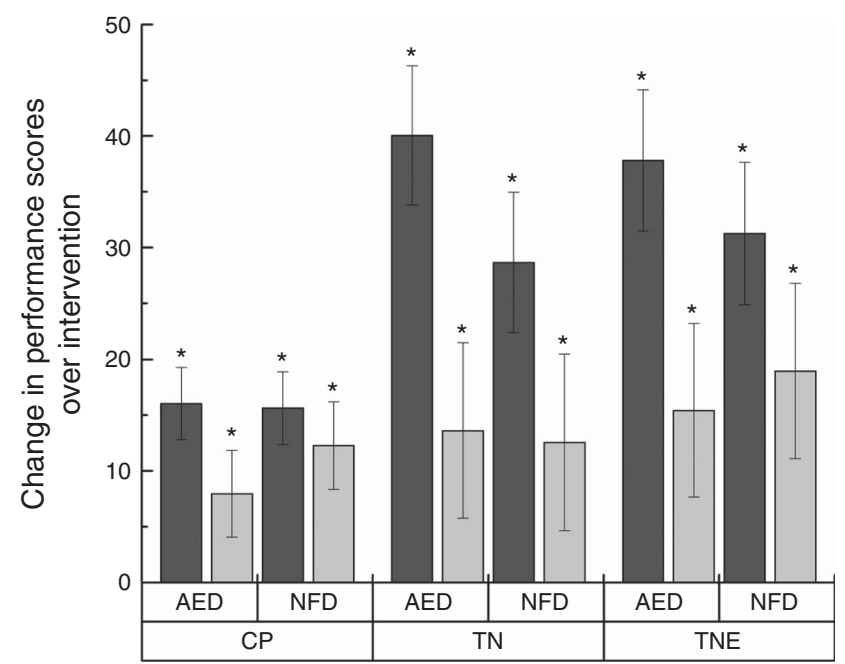

Fig. 6. Mean change in performance indices before and after the 12-week intervention in the almond-enriched diet (AED, $n$ 43) and the nut-free diet (NFD, $n$ 43) groups immediately after lunch $(\square)$ and $35 \mathrm{~min}$ after lunch ( $\square$ ) consumption. Values are means, with their standard errors obtained from a linear mixed-effects model with intervention period and lunch period as within-subject factors and intervention group and lunch group as betweensubject factors. * Significant differences for change in performance indices over the intervention period $(P<0.001)$. CP, concentration performance (total number of correct items marked minus errors of commission); $\mathrm{TN}$, quantitative performance (total number of items processed); TNE, qualitative performance (total number of items processed minus total errors).

An important finding of the study was the effect of lunch composition on cognitive function. Consumption of an almondenriched high-fat lunch reduced the decline in memory by $57.7 \%$ compared with the high-carbohydrate lunch. Because of the paucity of studies in this area, the clinical significance and practical implications of this acute change are yet to be established. This acute effect of almond consumption on memory may be attributable to their high fat and fibre content and lower carbohydrate content. Almond consumption has previously been shown to have a moderating effect on postprandial blood glucose concentrations ${ }^{(24,25)}$. Fat and fibre can lower postprandial glycaemia by delaying gastric emptying ${ }^{(26)}$ and intestinal transit time ${ }^{(27)}$, respectively. Lower than normal blood glucose concentrations impair cognitive function ${ }^{(28)}$. Hyperglycaemia has also been associated with impaired performance but primarily among individuals with diabetes ${ }^{(29)}$. In one study where memory was assessed over a period of $4 \mathrm{~h}$ after breakfast consumption, a low glycaemic index breakfast improved memory performance more than a high glycaemic index breakfast indicating that the rate of glucose release into the circulation may be responsible for these effects ${ }^{(30)}$. Consumption of almonds with a meal can reduce the glycaemic impact of carbohydrate, thereby maintaining optimum blood glucose concentrations for memory tasks.

In our study, almond consumption at lunch did not ameliorate the post-lunch dip in attention. However, a systematic review indicates that there are differences in how cognitive function modalities respond to changes in macronutrient compositions in healthy adults with no cognitive impairments ${ }^{(9)}$. Hence, the attention domain of cognitive function and tasks employed to assess attention may not be as sensitive to macronutrient manipulation compared with the memory domain ${ }^{(9)}$.

Dietary factors other than lunch composition can influence the post-lunch dip as well. Some researchers believe that the midday meal size (energy content of meal) may influence the decline in cognitive function after lunch. Larger lunches are associated with an increased number of errors in selective attention $^{(31)}$ and decreased cognitive performance in general ${ }^{(32)}$ compared with smaller lunches. In the present study, the amount of food given to participants was based on $23-25 \%$ of their estimated daily energy requirements. This is in line with customary intake levels in the population $^{(20)}$, so was ecologically valid though perhaps not a test of the extent of the effect. Others argue that the post-lunch dip is more attributable to endogenous rhythms ${ }^{(33)}$ or is a consequence of conditioned lunch effects ${ }^{(34)}$ rather than being causally linked to lunch composition or size. For example, some studies have observed differences in cognitive function between late morning and early afternoon even in individuals that had not eaten lunch ${ }^{(2,35)}$, but individuals who ate lunch had a greater postlunch dip in sustained attention compared with the lunch skippers $^{(2)}$. Although we did not control the time at which lunch was provided to participants (10.30-12.30 hours), there are limited data indicating that the post-lunch dip in attention and reaction to a new stimulus are not affected by the time at which lunch is eaten ${ }^{(2,35)}$. Other explanations for the post-lunch dip have been proposed including insulin surges ${ }^{(36)}$ increased cortisol $^{(37)}$ or serotonin concentrations ${ }^{(8)}$ among others.

This 12-week randomised weight loss trial improved memory and attention performance in overweight and obese energyrestricted adults. This is contrary to what has been observed in most ${ }^{(14-16)}$ but not all ${ }^{(18)}$ studies on dieting adults. The improvement in cognitive function could stem from the intervention itself with participants having undergone dietary counselling to make healthier choices and lose weight. The dieters in other studies $^{(14-16)}$ were not participating in structured weight loss interventions and may not have had access to dietary counselling. However, in spite of restricting the testing occasions to before and after the 12-week intervention, there is a possibility of practice effects $^{(38)}$. There were no additional improvements in memory and attention with almond supplementation. Although a previous nut-based trial has demonstrated improvements in working memory and speed of processing in energy-restricted adults consuming nut-supplemented diets ${ }^{(18)}$, this study did not have a control group. Hence, a comparison of the magnitude of improvements in cognitive function between a nut supplemented and a NFD could not be made. Another interventional study conducted in young but non-dieting adults observed an $11 \%$ increase in inferential verbal reasoning after consumption of $60 \mathrm{~g}$ walnuts/d for 8 weeks ${ }^{(39)}$ but observed no changes in non-verbal reasoning and memory scores.

Interestingly, weight loss is positively associated with cognitive decline in older adults ${ }^{(40)}$. Although this is contrary to the improved effects on cognitive function with weight loss observed in the present study, we did not exclusively recruit older people and our intervention may not have been long enough to observe these changes. Although the present study showed no effects of BMI (at baseline) on cognitive function, a recently published 
study observed a protective effect of obesity on cognitive function in people aged 45 years and older, particularly in women $^{(41)}$. The lack of an effect noted here may be attributable to the absence of normal-weight individuals for comparison. We also did not find any BMI, age or sex interaction effects on cognitive function. However, we did find a negative association between age and attention performance on the ' $\mathrm{d} 2$ ' test regardless of diet or lunch groups. Cognitive decline with ageing is a common occurrence ${ }^{(42)}$

The strength of the study lies in the novelty of investigating the acute effects of almond consumption on the post-lunch dip in cognitive function and the long-term effects of almond consumption on cognitive function in a weight loss intervention. A limitation of the study was lack of a power analysis on cognitive function outcomes, which may have led to undetectable intervention effects. In addition, the generalisability of the findings are limited to healthy, young to middle aged, overweight and obese adults with no cognitive function impairments who are participating in a weight loss intervention.

In conclusion, consumption of an almond-enriched high-fat lunch acutely ameliorated the post-lunch dip in memory and almond consumption at midday may be an effective means to maintain memory following the midday meal. However, almond consumption over 12 weeks did not further enhance the improvements in cognitive function outcomes with weight loss. Nevertheless, the literature indicates that almond consumption enhances satiety ${ }^{(43)}$ and reduces hunger and desire to eat ${ }^{(24)}$, properties that may minimise hunger-related thoughts that can acutely impair cognitive function in dieters ${ }^{(15)}$. Further research is needed to determine the replicability of the acute and long-term effects of almond consumption on cognitive function.

\section{Acknowledgements}

The authors thank the research team for their assistance in conducting the study.

The study was supported by the Almond Board of California (Modesto, CA, USA). The funders had no role in the study conception, design and implementation, data collection, data analysis or interpretation of results. R. D. M. received research support from the Almond Board of California.

The authors' responsibilities were as follows: J. D., S.-Y. T. and R. D. M.: conceived the study and shared equal responsibility in writing the manuscript and of its final content. All authors read and approved the final manuscript.

S.-Y. T. and J. D. had no conflicts of interest.

\section{References}

1. Craig A (1986) Acute effects of meals on perceptual and cognitive efficiency. Nutr Rev 44, 163-171.

2. Smith AP \& Miles C (1986) Effects of lunch on selective and sustained attention. Neuropsychobiology 16, 117-120.

3. Smith A, Leekam S, Ralph A, et al. (1988) The influence of meal composition on post-lunch changes in performance efficiency and mood. Appetite 10, 195-203.

4. Blake MJF (1967) Time of day effects on performance in a range of tasks. Psychon Sci $\mathbf{9}, 349-350$.
5. Hildebrandt G, Rohmert W \& Rutenfranz J (1974) 12 and $24 \mathrm{~h}$ Rhythms in error frequency of locomotive drivers and the influence of tiredness. In J Chronobiol 2, 175-180.

6. Smith A, Kendrick A, Maben A, et al. (1994) Effects of fat content, weight, and acceptability of the meal on postlunch changes in mood, performance, and cardiovascular function. Physiol Behav 55, 417-422.

7. Wells AS, Read NW \& Craig A (1995) Influences of dietary and intraduodenal lipid on alertness, mood, and sustained concentration. Br J Nutr 74, 115-123.

8. Spring B, Maller O, Wurtman J, et al. (1982) Effects of protein and carbohydrate meals on mood and performance: interactions with sex and age. J Psychiatr Res 17, 155-167.

9. Hoyland A, Lawton CL \& Dye L (2008) Acute effects of macronutrient manipulations on cognitive test performance in healthy young adults: a systematic research review. Neurosci Biobehav Rev 32, 72-85.

10. Nooyens ACJ, Bueno-de-Mesquita HB, van Boxtel MPJ, et al. (2011) Fruit and vegetable intake and cognitive decline in middle-aged men and women: the Doetinchem Cohort Study. Br J Nutr 106, 752-761.

11. Valls-Pedret C, Lamuela-Raventós RM, Medina-Remón A, et al. (2012) Polyphenol-rich foods in the Mediterranean diet are associated with better cognitive function in elderly subjects at high cardiovascular risk. I Alzheimers Dis 29 , 773-782.

12. O'Brien J, Okereke O, Devore E, et al. (2014) Long-term intake of nuts in relation to cognitive function in older women. J Nutr Health Aging 18, 496-502.

13. Barbour JA, Howe PRC, Buckley JD, et al. (2014) Nut consumption for vascular health and cognitive function. Nutr Res Rev 27, 131-158.

14. Green MW, Rogers PJ, Elliman NA, et al. (1994) Impairment of cognitive performance associated with dieting and high levels of dietary restraint. Physiol Behav 55, 447-452.

15. Green MW, Elliman NA \& Rogers PJ (1997) Impaired cognitive processing in dieters: failure of attention focus or resource capacity limitation? Br J Health Psychol 2, 259-267.

16. Green MW \& Rogers PJ (1998) Impairments in working memory associated with spontaneous dieting behaviour. Psychol Med 28, 1063-1070.

17. Vreugdenburg L, Bryan J \& Kemps E (2003) The effect of self-initiated weight-loss dieting on working memory: the role of preoccupying cognitions. Appetite 41, 291-300.

18. Halyburton AK, Brinkworth GD, Wilson CJ, et al. (2007) Low- and high-carbohydrate weight-loss diets have similar effects on mood but not cognitive performance. Am J Clin Nutr 86, 580-587.

19. Dhillon J, Tan S-Y \& Mattes RD (2016) Almond consumption during energy restriction lowers truncal fat and blood pressure in compliant overweight or obese adults. J Nutr 146, 2513-2519.

20. US Department of Agriculture \& US Department of Health and Human Services (2012) Lunch: percentages of selected nutrients contributed by food and beverages consumed at lunch, by gender and age: What We Eat in America, NHANES 2011-2012. http://www.ars.usda.gov/SP2UserFiles/ Place/80400530/pdf/1112/Table_17_LUN_GEN_11.pdf (accessed September 2012).

21. Benton D \& Parker PY (1998) Breakfast, blood glucose, and cognition. Am J Clin Nutr 67, 772S-778S.

22. Randolph C, Tierney MC, Mohr E, et al. (1998) The Repeatable Battery for the Assessment of Neuropsychological Status (RBANS): preliminary clinical validity. J Clin Exp Neuropsychol 20, 310-319.

23. Brickenkamp R \& Eric Z (1998) d2 Test of Attention. Boston, MA: Hogrefe publishers. 
24. Tan SY \& Mattes RD (2013) Appetitive, dietary and health effects of almonds consumed with meals or as snacks: a randomized, controlled trial. Eur J Clin Nutr 67, 1205-1214.

25. Josse AR, Kendall CWC, Augustin LSA, et al. (2007) Almonds and postprandial glycemia - a dose-response study. Metabolism 56, 400-404.

26. Lin HC, Zhao XT \& Wang L (1996) Jejunal brake: inhibition of intestinal transit by fat in the proximal small intestine. Dig Dis Sci 41, 326-329.

27. Salas-Salvadó J, Bulló M, Pérez-Heras A, et al. (2006) Dietary fibre, nuts and cardiovascular diseases. Br J Nutr 96, S46-S51.

28. Fruehwald-Schultes B, Born J, Kern W, et al. (2000) Adaptation of cognitive function to hypoglycemia in healthy men. Diabetes Care 23, 1059-1066.

29. Cox DJ, Kovatchev BP, Gonder-Frederick LA, et al. (2005) Relationships between hyperglycemia and cognitive performance among adults with type 1 and type 2 diabetes. Diabetes Care 28, 71-77.

30. Benton D, Ruffin M-P, Lassel T, et al. (2002) The delivery rate of dietary carbohydrates affects cognitive performance in both rats and humans. Psychopharmacology (Berl) 166, 86-90

31. Smith A, Ralph A \& McNeill G (1991) Influences of meal size on post-lunch changes in performance efficiency, mood, and cardiovascular function. Appetite 16, 85-91.

32. Craig A \& Richardson E (1989) Effects of experimental and habitual lunch-size on performance, arousal, hunger and mood. Int Arch Occup Environ Health 61, 313-319.

33. Hildebrandt G, Rohmert W \& Rutenfranz J (1974) 12 and 24 h Rhythms in error frequency of locomotive drivers and the influence of tiredness. Int J Chronobiol 2, 175-180.
34. Colquhoun WP (1971) Circadian variations in mental efficiency (Circadian rhythms in human mental performance from waking day, round of clock and simulated shift work studies). In Biological Rhythms and Human Performance, pp. 39-107 [WP Colquhoun, editor]. London and New York: Academic Press.

35. Smith AP \& Miles C (1986) The effects of lunch on cognitive vigilance tasks. Ergonomics 29, 1251-1261.

36. Woods SC \& Porte D (1974) Neural control of the endocrine pancreas. Physiol Rev 54, 596-619.

37. Kirschbaum C, Wolf OT, May M, et al. (1996) Stress- and treatment-induced elevations of cortisol levels associated with impaired declarative memory in healthy adults. Life Sci $\mathbf{5 8}$, $1475-1483$

38. McCaffrey RJ \& Westervelt HJ (1995) Issues associated with repeated neuropsychological assessments. Neuropsychol Rev 5, 203-221.

39. Pribis P, Bailey RN, Russell AA, et al. (2012) Effects of walnut consumption on cognitive performance in young adults. $\mathrm{Br} \mathrm{J}$ Nutr 107, 1393-1401.

40. Suemoto CK, Gilsanz P, Mayeda ER, et al. (2015) Body mass index and cognitive function: the potential for reverse causation. Int J Obes 39, 1383-1389.

41. Kim S, Kim Y \& Park SM (2016) Body mass index and decline of cognitive function. PLOS ONE 11, e0148908.

42. Deary IJ, Corley J, Gow AJ, et al. (2009) Age-associated cognitive decline. Br Med Bull 92, 135-152.

43. Hull S, Re R, Chambers L, et al. (2015) A mid-morning snack of almonds generates satiety and appropriate adjustment of subsequent food intake in healthy women. Eur J Nutr $\mathbf{5 4}$, 803-810. 